\title{
Leo Spitzer censeur militaire
}

Résumé: Leo Spitzer, encore un jeune homme, a dirigé un des bureaux de la censure militaire autrichienne à Vienne pendant la 1 $1^{\text {ère }}$ Guerre mondiale. Son travail consistait, entre autres, à éliminer dans les lettres des prisonniers italiens à leur famille toute mention de la faim que ceux-ci enduraient, afin de préserver l'image de l'Autriche-Hongrie. Philologue, il en a profité pour rassembler en un livre toutes les périphrases et allusions, en italien et en dialecte, utilisées par les prisonniers pour demander à leur famille l'envoi de colis alimentaires. Ce faisant, il semble ne pas s'être interrogé sur la dimension "éthique » de son comportement. Reste un livre important de lexicologie : Die Umschreibungen des Begriffes „Hunger“ im Italienischen.

Mots clés: Censure, éthique, philologie, prisonniers, faim.

Leo Spitzer a passé la $1^{\text {ère }}$ Guerre mondiale à Vienne, dans les bureaux de la censure militaire.

Le jeune Privat-Dozent (28 ans en 1915), habilité depuis 1912 avec une thèse sur le verbe « échaler » (« cueillir les noix ») en français médiéval, s'était vu confier la responsabilité d'un des cinq bureaux chargés de lire et de censurer le courrier expédié par les cent mille prisonniers de guerre italiens qui se trouvaient internés dans les camps de la Double Monarchie. Il s'agissait pour les censeurs de repérer, puis de supprimer, tous les passages de ces lettres pouvant nuire à l'image de l'Autriche-Hongrie. Il était en particulier interdit aux prisonniers d'évoquer la faim, car les pays belligérants étaient tenus par les conventions internationales d'assurer aux prisonniers de guerre une alimentation correcte. Spitzer a donc bénéficié d'une véritable «manne philologique », car il a eu entre les mains des milliers de lettres de prisonniers qui tentaient d'alerter par tous les moyens linguistiques leurs familles en Italie sur la nécessité de leur envoyer des colis. Il en est résulté un livre de 350 pages denses, qu'il publia en 1920 : Die Umschreibungen des Begriffes „Hunger“ im Italienischen [Les périphrases du concept de « faim » en italien] (Spitzer 1920). Comme il l'écrit dans sa préface : « L'organisation de bureaux de la censure et l'effort adverse pour s'exprimer de manière secrète a constitué au niveau philologique une expérience qui n’aurait jamais pu être menée à une telle échelle dans un autre contexte. » (cité par Gumbrecht 2002, 95¹). Nous nous

1 Nous traduisons de l'allemand toutes les citations du livre de Spitzer.

2 Open Access. (C) 2021 Robert Kahn, published by De Gruyter. (c) BY-NC-ND This work is licensed under the Creative Commons Attribution-NonCommercial-NoDerivatives 4.0 International License. 
proposons dans un premier temps de donner quelques exemples de ces résultats scientifiques : le style et la méthode du grand Leo Spitzer sont bien présents dès cette publication de jeunesse. Puis nous nous intéresserons à quelques phases de cette sorte de duel qui eut lieu entre les prisonniers de guerre et Spitzer en tant que chef de la censure, et enfin nous nous autoriserons quelques remarques à portée « éthique », en particulier sur le rapport entre « science » et « pouvoir ».

\section{Le corpus}

Le livre constitue un recueil de milliers de citations authentiques, prélevées par Spitzer avant le retour des lettres dans le circuit postal. Toutes sont référencées par le lieu d'émission et celui de destination. Le philologue repère et classe 21 grands types ou motifs, ce qui peut paraître peu, mais autorise d'infinies variations. Citons quelques-uns de ses motifs et techniques : l'abréviation, «f. » pour fame ; l'anagramme : «la signora Emaf »; l'euphémisme : " apetito »; les attributs personnifiés : la « signora », la "sorcière », la « brune », la « fiancée autrichienne », la « grande ${ }^{2} »:$ "Venise ${ }^{3}$ : Di salute sto benissimo ma la granda aumenta » (Spitzer 1920, 43). "L'air, le vent » offrent des possibilités synonymiques : la cure d'air est une allusion ironique au proverbe « on ne vit pas seulement d'air ». D’où : " Côme : L'aria qui a Mauthausen è finissima, ma lappettito è ancora più fina che l'aria, perciò comprenderete che di sola aria fin non si può vivere, e questo è già un vecchio proverbio di Mauthausen » (Spitzer 1920, 55). Ou encore l'évocation des saints, qui constitue pour les prisonniers une mine quasiment inépuisable, mais qui ne déroute pas Spitzer. Sainte Lucie est une figure majeure en Italie. Pour le censeur, plusieurs raisons peuvent expliquer que les prisonniers utilisent son image pour envoyer leur message crypté : elle aurait envoyé après sa mort des navires chargés de blé vers des rivages souffrant de la famine, elle était la patronne des pauvres, mais, surtout, sa fête était précédée d'un jour de jeûne. D’où : «Palerme : qui ogni giorno è S. Lucia ed ogni giorno si festeggia con vigilia, ti basta questo » (Spitzer 1920, 112). Bien entendu sont évoqués les états corporels : "se serrer la ceinture » = « si tira la cinghia » (77), le " chant » de l'estomac devient le chant d'un oiseau : « ed ogni giorno ci divertiamo a sentire il canto del Osignuolo. » (137). Et différents animaux peuvent porter le message secret : la cigale, comme dans la fable, ou la grenouille, ou, surtout, le chameau,

2 Les termes en français sont traduits par nous depuis l'allemand.

3 Les indications de lieux sont en italien dans l'original. Nous les traduisons. 
le symbole de la frugalité, parfois rencontré en Libye, colonie italienne, et à qui il arrive toutes sortes d'aventures : "Bergame : io sto bene di salute e di tutto. Solo chè cui otrovato il amico Camello il marito della Scaiusa. » (194). Nous reparlerons du chameau. Une des rares activités possibles dans le camp, la lecture, peut dissimuler le message secret, comme dans cette lettre pour Trapani : « mi levo la fame quando non si puo leggo il giornale (capite ?)» (155). Évidemment, l'admonestation entre parenthèses est d'une grande maladresse, signalant ce que le scripteur souhaite dissimuler. Le nom d'un prisonnier de Mauthausen qui écrit à Livourne n’a pas dû non plus tromper le lecteur : Umberto Moltafame (19). De façon générale, Spitzer a pu relever un grand nombre de tentatives placées en dehors du corps même de la lettre, dans l'adresse postale, la signature, la date : Via Fame, Olomutz I Genaio anno della fame 1917 (239). Le censeur-philologue a ainsi réussi, à partir de cette collecte effectuée de 1915 à 1918, à établir une véritable encyclopédie d'expressions, locutions, périphrases et autres expédients linguistiques destinés à signifier à la famille, aux amis, la situation réelle, la souffrance des prisonniers. Pour ce faire, pour identifier, analyser, classer cette masse de données, Spitzer utilise les méthodes que lui ont enseignées ses maîtres Meyer-Lübke et Karl Vossler : haute érudition, connaissances linguistiques (il a vécu un an en Italie, et il y mourra d'ailleurs en 1960, près de Rome) ; utilisation des travaux des pairs : Albert Dauzat, L’argot des tranchées, par exemple, et plus généralement, toutes les études sur l'argot et les langages spécialisés en français et en italien, comme par exemple Marcel Cohen, Le langage de l'École Polytechnique, Niceforo, Le génie de l'argot - et donc il participe au débat : les trouvailles des prisonniers relèvent-elles de la création collective ou du talent individuel ? Spitzer dialogue ici avec Sainéan et Marcel Schwob. Bien entendu, les notes de bas de page spitzériennes sont très nombreuses et précises. Nous ne pouvons donner ici qu'un très bref aperçu de sa moisson, et nous terminerons cette évocation du corpus par deux sous-motifs « littéraires » particulièrement intéressants.

Le motif dantesque d'abord. Le comte Ugolino, au chant XXXIII de l'Enfer, raconte au poète comment, prisonnier dans la tour de la Mue, il a mangé ses enfants morts. Les prisonniers pensaient sans doute, à tort en ce qui concerne Spitzer, que leurs censeurs n'étaient pas de grands connaisseurs de la littérature italienne. D’où une abondance de références dans les lettres et cartes, d'après Spitzer il s'agit du motif littéraire le plus représenté dans son corpus. La première occurrence date de 1915, au camp de Mauthausen ; Spitzer évoque ensuite une véritable « épidémie » (Spitzer 1920, 160) qui cessera quand les prisonniers se rendront compte, d'après l'absence de réaction de leurs familles qui n'envoyèrent pas de colis, que l'allusion avait été décryptée par la censure. Citons le censeur en chef, Leo Spitzer : « À la fin de la guerre une allusion à Ugolino représentait une rareté - une preuve des fluctuations dans la cote d'amour d'une trouvaille indi- 
viduelle» (Spitzer 1920,161). Voici quelques exemples : «Venise : se si va avanti sempre cosi finiro come Ugolino di Francia pasenzia » («Francia » [France] était une façon géographique de dire « faim »). «Palerme : Fra gli amici comuni si trova con me Ugolino, il nostro, collega del primo corso liceale. » (ibid.).

Spitzer commente : «Ugolino est prisonnier comme nous » veut bien sûr dire : "Ici règne la faim ». " Milan : mi trovo contento tanto più che qui hoffato la conoscenza con un certo conte ugolino di pisa » (Spitzer 1920, 162). Et encore : « Pesaro : Saluta per me tutta la familia Ugolini ». Ou bien le scripteur se qualifie-t-il luimême : « Naples, signature de la lettre : "Ugolino la Vittima" ». Spitzer explique aussi que les initiales C.U pour Conte Ugolino ne trompent évidemment aucun censeur sérieux. Cette utilisation de la référence culturelle amène lesdits censeurs à créer eux-mêmes un néologisme : rimediare Dante [éliminer une allusion à Ugolino]. Nous reviendrons sur cet aspect de « duel », d'ailleurs très inégal, entre les fonctionnaires et les malheureux prisonniers. Ceux-ci, dans leur détresse, ont fait fond sur le symbole de la culture et de la littérature italienne, mais en négligeant le fait que Dante est aussi un bien commun de l'humanité, fait qui en l'occurrence se retourne contre eux. Au début du XXe siècle, quelques-uns des plus grands spécialistes de Dante, collègues de Spitzer, étaient de langue allemande : Erich Auerbach, Karl Vossler. De toutes façons, connaître la terrible histoire du comte n'exigeait pas une très grande érudition.

Dans le très riche catalogue spitzérien, nous avons choisi d'isoler un dernier type ou motif, pour une fascinante raison de coïncidence littéraire : il s'agit de l'évocation, par les prisonniers de guerre, de ces " artistes de la faim », qui ont réellement exercé leur art en Europe jusqu'au début du XXe siècle. L'un d'entre eux apparaît dans le recueil de Spitzer. Il s'agit d'un certain Succi : « En Italie du Nord et en Italie centrale les réminiscences de Succi ont bénéficié de la même prédilection que l'Ugolino de Dante. » Ce " performeur », comme on dirait aujourd'hui, était célèbre pour avoir, avec l'aide d'une liqueur opiacée, jeûné du 18 août au 17 septembre 1886 à Milan (Spitzer 1920, 53-54). À propos d'un autre artiste célèbre, Merlatti, Spitzer cite en note de bas de page l'article « Hunger » [faim] du dictionnaire Brockhaus : «À la fin du jeûne son corps s'était complètement ratatiné, les mains et les pieds apparaissaient d'une longueur inhabituelle, le visage était extraordinairement émacié, le nez remarquablement pointu » (Spitzer 1920, 81). Voici quelques exemples du thème dans des lettres de prisonniers : « Turin : sto molto bene e per fortuna mia, ho lasciato la Cura Succi». « Florence: sono sempre stato in compagnia dell'amico Succi, non però col Succi di Monticelli, spero che avrete capitò ». " Cuneo : qui abbiamo una forta bava di Levante, e li facciamo concorenza al notto Succi » (Spitzer 1920, 54-55). Les prisonniers pensaient sans doute, encore une fois à tort, que la renommée de cet artiste n'avait pas franchi les Alpes. Ce qui nous a fasciné dans cet exemple, collecté avant novembre 1918, 
est la rencontre possible dans l'esprit du lecteur avec l'un des textes de fiction les plus célèbres de la première moitié du XX ${ }^{\mathrm{e}}$ siècle, Der Hungerkünstler [L’Artiste de la faim], publié en 1922 par Franz Kafka. On oublie toujours à quel point les textes kafkaïens sont ancrés dans le réel. Mais ils témoignent en général d'une pitié pour les «frères humains » qui nous a semblé faire défaut à Leo Spitzer. Celui-ci s'est surtout intéressé à la dimension ludique, ou « sportive », de sa tâche de censeur. Il a souhaité très vivement être un « bon censeur en chef », capable de neutraliser toutes les astuces et ruses des prisonniers de guerre. Il s'agit maintenant de s'intéresser à cet aspect pragmatique d'une sorte de « duel ».

\section{Pragmatique du duel}

Spitzer, au début de l'ouvrage (Spitzer 1920, 4), donne quelques indications précises sur la façon dont s'organisait son travail et celui de ses subordonnés, puisqu'il était un " superviseur », ou, comme il se qualifie lui-même, un " hypercenseur ». Les lettres et cartes étaient lues une première fois, les passages soupçonnés étaient soulignés en rouge. Ensuite, elles étaient relues par le superviseur, donc Spitzer, à qui revenait la décision finale. La censure signifiait concrètement rendre illisibles les passages définitivement condamnés, par recours à l'encre noire. Ensuite, les lettres retournaient dans le circuit d'expédition ou, dans les cas les plus graves, étaient détruites. Ce processus n'a subi aucune critique de la part de Spitzer, on y reviendra. C'est un travail proche de celui qu'effectue le malheureux Winston dans 1984 de George Orwell. Les prisonniers quant à eux s'évertuent à faire preuve d'imagination. Par exemple, l'expression Kriegsgefangenenlager [camp de prisonniers de guerre] se retrouvait orthographiée « Krist chefamen lader » [italien : Cristo, che fame di ladro] (Spitzer 1920, 158-159). Mais il se trouve que le Corriere della Sera avait divulgué cette trouvaille, et ce journal était lu à Vienne. Cependant il arrive que le censeur de base laisse passer certaines formulations : une lettre est adressée à Londres à l'entreprise Crep \& Defin [Crepo di fame], que seul l'hypercenseur, c'est-à-dire Leo Spitzer, identifie (Spitzer 1920, 68). Un des procédés qui pose le plus de problèmes à ses subordonnés est le recours à des expressions locales et/ou argotiques, comme $\operatorname{artibi}(a)$ pour «pain » (Spitzer 1920, 229). En de telles occurrences il faut toute la science de l'érudit pour remediare, c'est-à-dire censurer avec efficacité. Spitzer le dit d'ailleurs : ce travail, pour lui comme pour toute son équipe, développe les qualités d'analyse des textes qui caractérisent les méthodes philologiques et herméneutiques. En définitive, la « victoire ", écrit Spitzer, ne peut revenir aux prisonniers (Spitzer 1920, 262). La meilleure preuve en est que, dans certains courriers, les prisonniers s'adressent directement à la 
censure pour exprimer leur colère, pour se plaindre de la faim et invectiver l'Autriche, l'Empereur, le chef du camp, les censeurs, etc. Par exemple cette lettre envoyée à Parme : Prego la Censura di compatire il mio male scritto perche il motivo viene dalla fabrica dela appettito. Prego di fare pervenire la presente che tali sono privi di notizie. (Spitzer 1920, 259). L'exemple qui nous a semblé le plus subtil, d'ailleurs reconnu comme tel par Spitzer, bien qu'il ait, une fois de plus déjoué la ruse, fait intervenir ce " chameau » déjà rencontré : "Crova Vercellese : avrei molte cose da narrarle, ma, come Ella sa, è più facile che passi un camello per la cruna d'un ago anziche sfuggano al microscopio dei signori censori certe noterelle » (Spitzer 1920, 272). Spitzer commente : au moment où le scripteur feint de louer la perspicacité de la censure, puisqu'il est plus difficile de la tromper que pour un chameau de passer par le chas d'une aiguille, la présence dans la citation biblique du chameau est une allusion à la faim, qui évidemment ne trompe pas non plus l'hypercenseur, celui qui ne s'en laisse pas conter.

Mais c'est justement tout le problème. Spitzer ne laisse rien passer, ce qui nous semble poser un problème éthique.

\section{Une éthique de la censure est-elle possible ?}

Vers la fin du livre, Spitzer commente les « erreurs » des prisonniers : beaucoup pensent que les censeurs ne savent pas l'italien et qu'ils déchirent les lettres de façon arbitraire, ou alors que bien des finesses ou des localismes leur échappent, ou encore qu'ils procèdent surtout par sondage, sans lire l'intégralité de la carte ou de la lettre, comme l'exigent les instructions officielles. Et c'est là que l'hypercenseur rédige une parenthèse qui en dit long : « (on ne peut taire le fait que des censeurs médiocres ont pu se croire de temps à autre dispensés de cette règle de base et qu'ils ont pu ainsi nuire gravement à l'État en bousillant leur travail) ${ }^{4}$ ».

L'idée est donc que la tâche de la censure est très importante, et que la fidélité à cet État qui a pourtant cessé d'exister au moment de la publication du livre prime sur ce qu'il faut bien appeler le « devoir d'humanité ». Il faut en effet rappeler, avec des historiens comme Michel Ostenc, quelle était la situation réelle des prisonniers italiens dans les camps de la double monarchie ( $c f$. Ostenc 2014). Michel Ostenc explique, par exemple, que dans le camp de Milowitz, près de 10000 prisonniers sur 14000 moururent pendant les 4 à 5 mois qui suivirent la

4 « minderwertige Zensoren [...] schädigten schwer den Staat durch ihre Hudelarbeit. » (Spitzer 1920, 259). 
défaite de Caporetto. Il rappelle que le grand écrivain Carlo Emilio Gadda, qui fut l'un de ces prisonniers de guerre, fut hanté dans ses cauchemars par le souvenir de la faim pendant des années. Il évoque le cas d'un malheureux dont l'on retrouva le cadavre avec de l'herbe dans la bouche. L'historien conclut : « Les observateurs découvrirent après l'armistice des détenus squelettiques, affamés et en haillons » (Ostenc 2014, 35).

On peut donc s'étonner de l'ignorance, au mieux, ou du cynisme de Spitzer, qui écrit en préambule de son livre : «Le fait est que les prisonniers de guerre italiens en Autriche-Hongrie ont infiniment souffert de la faim : ils recevaient les mêmes rations (et la même solde) que nos propres soldats et ainsi tout est dit " (Spitzer 1920, 8). Il rend d'ailleurs le blocus des Alliés responsable de la situation alimentaire dans les camps de prisonniers. L'enjeu de l'acte de censure n'est donc pas seulement de permettre à de jeunes philologues de s'exercer à la pratique de l'érudition et de l'herméneutique : le fait de recevoir ou non un colis alimentaire envoyé par la famille ou les amis pouvaient changer un destin. Or, du fait du travail acharné, " scientifique », de Leo Spitzer et de son équipe, bien des colis n’ont jamais été expédiés, tous ceux, par exemple, qui étaient demandés par une allusion au comte Ugolino. Spitzer a d'ailleurs rédigé, à l'intention de toutes les équipes qui traitaient les courriers en italien, une sorte de mémento qui a dû se révéler très utile, puisque plus d'un de ses collaborateurs n'avait pas saisi, par exemple, la signification du « chameau ».

Mais s’agit-il bien du même Leo Spitzer qui avait rédigé L’Anti-Chamberlain et ce beau livre, qu'Agnès Steuckardt qualifie, peut-être un peu vite, de « pamphlet pacifiste », Fremdwörterhatz und Fremdvölkerhaß (Spitzer 2013) ${ }^{5}$, cette belle polémique contre la tentative, ridicule, initiée par la puissante «Association de la langue allemande » de " nettoyer » ces impuretés que représentent les emprunts à des langues étrangères ? Spitzer montre par exemple que le remplacement, à Vienne de Sauce par Tunke consisterait à évincer un mot devenu courant par un mot d'emprunt, appartenant en fait à un dialecte étranger, le germano-prussien (Spitzer 2013, 83). Et il conclut magistralement : " Pour tous ces nettoyeurs, le mot étranger est une "souillure" de la langue maternelle, et ainsi ils ne pensent pas non plus que si péché il y a, c’est le péché originel de la langue humaine, qui toujours est “mélangée” et qui l'est nécessairement » (Spitzer 2013, 90). Cette analyse, brillante, annonce le travail de Klemperer ou d'Orwell. Alors pourquoi cette traque des périphrases du mot « faim » en italien dans un bureau du ministère à Vienne ? On ne peut que se livrer à des conjectures : par patriotisme, c'est évident, et plaisir du jeu, de la chasse : les astuces des scripteurs italiens ont été

5 La citation d'A. Steuckardt se trouve p. 18. 
déjouées par l'équipe de Spitzer, mais aussi, peut-être, un sentiment plus obscur : le livre sur Chamberlain, et celui sur la « traque des mots étrangers », parus peu avant, n’ont pas dû faire que des amis à Spitzer dans le monde universitaire germanophone. Ce monument voué au travail de la censure impériale rétablirait un peu l'équilibre. Hans Ulrich Gumbrecht rappelle que Spitzer, après son habilitation, a dû attendre douze ans, une éternité, avant d'être enfin nommé professeur ordinaire à Marbourg ( $c f$. Gumbrecht 2002, 98-99), et qu'il a beaucoup souffert de cette situation. Une certaine tendance actuelle de la recherche en littérature comparée tend à opposer les deux « collègues », Leo Spitzer et Erich Auerbach ${ }^{6}$, en arguant du fait qu'à Istanbul, Spitzer se serait davantage intéressé à la langue et à la littérature turques que ne l'a fait l'auteur de Mimésis, et que donc il serait plus à même d'inspirer les travaux actuels, sensibles à la différence des cultures dans le cadre de la "mondialisation ». Mais cette opinion gagnerait peut-être à tenir davantage compte du contexte difficile des années 1920, et à ne pas réduire la grande complexité de la personnalité de Leo Spitzer à une seule dimension. À tout le moins, en accomplissant à la perfection son travail d'hypercenseur, le philologue viennois s'est dispensé de compassion. Un certain « laxisme » de son bureau aurait sans doute facilité la vie des prisonniers. Il n'en reste pas moins que, tel qu'il existe, le livre est aussi un très bel hommage ${ }^{7}$ rendu à la créativité et à l'imagination des locuteurs italiens et à leur langue.

\section{Bibliographie}

Gumbrecht, Hans Ulrich. Vom Leben und Sterben der großen Romanisten. Munich et Vienne : Carl Hanser, 2002.

Ostenc, Michel. « Les prisonniers italiens de la 1ère Guerre mondiale ». Guerres mondiales et conflits contemporains, $\mathrm{n}^{\circ}$ 252. Paris : PUF, 2014. 27-41.

Spitzer, Leo. Die Umschreibungen des Begriffes „Hunger“ im Italienischen. Halle : Max Niemeyer, 1920.

Spitzer, Leo. Traque des mots étrangers, haine des peuples étrangers. Jean-Jacques Briu (trad.). Limoges : Lambert-Lucas, 2013 [original : Fremdwörterhatz und Fremdvölkerhaß. Vienne : Manzsche Hof-, Verlags- und Universitäts-Buchhandlung, 1918].

\footnotetext{
6 Auerbach a, quant à lui, été sérieusement blessé à la jambe dans les tranchées.

7 La qualité du livre a été reconnue par des lecteurs imprévus : l'exemplaire sur lequel nous avons travaillé, celui de la Bibliothèque Nationale Universitaire de Strasbourg, a été estampillé, sans doute en 1942, de la croix gammée. Le bibliothécaire de l'époque pendant laquelle Strasbourg était une université « allemande » a, de toute évidence, renoncé à « désherber » un livre rédigé pourtant par un universitaire juif.
} 
Robert Kahn was an alumnus of the École Normale Supérieure de Saint-Cloud and a former senior lecturer of comparative literature at the University of Rouen. He is the author of Images, passages, Marcel Proust et Walter Benjamin (1996), of several essays on Proust, Benjamin, Auerbach, Perec, Sebald etc., a co-author of Poétiques du récit d'enfance, (2012), and a co-editor of La Retraduction (2010). He has translated works by Erich Auerbach, Walter Benjamin, and Franz Kafka. He passed away in April 2020, during the preparation of this volume. 
